\title{
Érik Canuel, transcréateur : Le Survenant (2005) et autres films
}

\section{Entretien accordé à Marie Pascal, Université Dalhousie}

Après avoir accompagné son père sur des tournages depuis son plus jeune âge, Érik Canuel a commencé la réalisation à vingt ans. Il a adapté plusieurs textes littéraires québécois (des romans avec Le Survenant (2005), Cadavres (2009), Lac Mystère (2013) ; une autobiographie avec Le dernier tunnel (2004) et une pièce de théâtre avec Barrymore (2011). Son film emblématique est Bon Cop, Bad Cop (2006) avec l'acteur Patrick Huard. Le réalisateur est également connu pour de nombreux clips de musique et des émissions TV. Collectionneur de bandes-dessinées et féru de musique, Canuel a pour objectif de mettre en valeur les arts les uns grâce aux autres afin de faire résonner son public, comme si chacun d'entre nous était un instrument de musique. Je l'ai rencontré dans un café de Montréal où, très généreux de son temps, il m'a conté sa réflexion sur ses adaptations cinématographiques, notamment $L e$ Survenant.

\section{Concernant le projet d'adapter un classique}

\section{M.P. Pourquoi avoir choisi Le Survenant ?}

E.K. Ce choix est avant tout en lien avec mon père. Mon père a grandi dans le Bas-Pays et c'est un gars qui aimait beaucoup cette littérature-là et cet esprit de vie. C'était quelqu'un qui, souvent, me disait dans mon jeune âge : "Tu devrais t'intéresser aux œuvres québécoises... » Le Survenant, tout le monde l'avait lu à l'école sauf moi ! Quand on me l'a proposé, ça a donc été d'abord par amour pour mon père qui était alors décédé mais ça a aussi été pour l'amour qu'il portait à la terre, à ce paysan-là, à ce bonhomme-là [Canuel fait ici référence à l'un des protagonistes du roman, le père Didace (joué par Gilles Renaud), habitant qui accueille le Survenant]. Mon père était un homme très ancré dans ces valeurs tout en étant également un vagabond du plaisir. C'était un homme qui était très libéral et qui n'avait pas d'œillères comme la majorité des gens, dans le roman, tous ceux qui entourent le Survenant. Évidemment, le roman m'a aussi beaucoup touché parce que c'est un récit qui se prête à démêler tous les tabous. Comme je disais à quelqu'un, ça aurait pu être l'histoire d'un Noir qui s'en vient au Québec, aujourd'hui, et qui vit la même espèce de rejet, la même exclusion, malgré tout ce qu'il pourrait apporter par son vécu. Cette espèce de dichotomie entre le personnage principal et ceux qui l'entourent pourrait être racontée à toutes les époques, dans tous les milieux. C'est pourquoi ce sujet m'interpelle beaucoup et aussi, ce personnage, amoureux de la liberté et de la vie, désireux de connaître les gens, qui se trouve confronté à des barrières sociales autant que géographiques. C'est aussi pour cette raison que j'ai accepté le projet.

\section{M.P. Quelle est votre relation, plus stylistique, avec ce classique de Germaine Guèvremont ?}

E.K. Devant un écrit, je fais toujours le choix de le lire puis de l'oublier le plus rapidement possible afin de me fier au scénario. Il faut que tu te sépares de l'œuvre littéraire parce que sinon, tu n'arriveras jamais à la justifier, à lui donner les lettres de noblesse qui lui reviennent. J'aime mieux m'approprier le roman et ne garder que ce qu'il m'a insufflé comme inspiration, pour en faire ma propre lecture cinématographique. Avec l'auteur du scénario, ça a été super. On a eu un très bon rapport mais j'ai souvent demandé des modifications et des changements quand je sentais que la teneur allait être, à certains moments, trop didactique, trop près du roman, trop fidèle. Je pense que le film est assez fidèle - je le crois d'après ce qu'on m'en a dit. Pour moi, ce que le roman amène à lire, c'est cette 
ode, cette poésie du langage et de la nature. La poésie du patois des gens et de cette vie de campagne. Le rêve qui se trouve dans la possibilité de voir autre chose, de vivre ce que le Survenant [joué par Jean-Nicolas Verreault] apporte à Angélina [jouée par Anick Lemay], elle qui est vouée à mener une vie de misère avec le fils du voisin. Mais il faut aussi parler de la fermeture d'esprit devant cette vie-là, devant cette liberté. C'est l'exemple du fils du bonhomme [le fils de Didace Beauchemin, Amable, joué par François Chénier], qui est aigri, fermé, méchant, jaloux, qui a peur de se faire voler ce que l'autre n'a jamais prétendu lui voler (terre, biens, affection de son père). C'est à cause de son aveuglement qu'il perd l'amour de son père, pas à cause du Survenant. En gros, ce que j'ai fait, c'est dans l'espoir de respecter l'écrit dans son inspiration et dans son souffle. J'aurais pu le traiter de façon plus froide, plus sobre, naturaliste presque. Non, j'avais besoin d'insuffler la beauté, le souffle de Dieu. Je ne suis pas nécessairement religieux ni pratiquant mais c'est essentiel d'apporter le souffle de l'univers, la beauté de la nature, de ce qui nous entoure et de ce qu'on peut être. Faire une œuvre poétique dans une espèce de chant, d'allégorie, tout en étant, en même temps, un hommage à mon père.

\section{M.P. Moi je trouve que dès le générique, il y a un souffle d'espoir.}

E.K. J'ai travaillé avec Frederic Back pour le générique. Il avait fait un film splendide en animation qui s'appelle L'homme qui plantait des arbres. Cette animation, les thèmes de mon film, et le fait que mon père ait planté 40000 arbres dans notre propriété à la campagne, ça revient au même. Cette poésie-là est évidente quand tu regardes L'homme qui plantait des arbres. C'est une tellement belle ode à la nature et à ce que l'homme peut faire... J'ai adoré m'en servir de générique, car ce style permet non seulement une ouverture sur le Survenant mais aussi, comme ces images-là sont comme des sérigraphies d'époque, c'est une ouverture sur un temps révolu. Difficile de ne pas sombrer dans le naturalisme et dans une espèce de côté très harsh, d'essayer de faire plus vrai que vrai tout le temps... C'est pour peindre de la lumière, en donner dans le cœur des gens malgré le drame épouvantable de ce roman, le drame d'un père qui perd celui qui a la place, dans son cœur, d'un fils. Une grande peine d'amour.

\section{M.P. Sur quels critères vous êtes-vous basé pour choisir l'acteur [Jean-Nicolas Verreault] qui allait jouer le rôle ? Êtes-vous satisfait du résultat ?}

E.K. C'est un acteur que j'aimais déjà beaucoup, que j'avais rencontré dans un des premiers shows de télévision que j'avais faits, il y a longtemps. C'est un bonhomme que j'aimais bien, que je trouvais fort sympathique, avec cet aspect un peu déglingué. J'aimais aussi le fait qu'il ait l'air d'un jeune premier pas parfait, juste un peu déjanté, juste assez différent pour avoir une stature similaire à Roy Dupuis mais sans cette espèce de perfection dans l'incarnation. Jean-Nicolas a un charisme tellement vrai ! Le rire, par exemple, c'est vraiment lui ! Lui, il n'était pas sûr mais moi, je lui disais : «Faut que ça éclate ! Faut dire que j'avais beaucoup aimé travailler avec lui dans La loi du cochon. Et à l'époque, des gars comme ça, de cet âgelà, avec ces caractéristiques-là... il n'y avait pas grand monde que je connaissais qui pouvait incarner le Survenant. C'est sûr qu'on peut faire bien des choses au cinéma mais tu mets pas Tom Cruise pour le Survenant. C'était Jean-Nicolas.

\section{Questions sur des scènes particulières}

E.K. Avant de répondre à tes questions sur le film, j'aimerais dire que c'est le roman qui m'a choisi. Moi, à la lecture, j'ai jamais considéré que c'était une grande œuvre mais j'ai toujours pensé que c'était une œuvre importante. L'œuvre est importante à un point qu'il fallait la redire, la ramener au goût du jour pour que les jeunes, les générations à venir, puissent 
s'abreuver de ce que j'appelle la « vérité de la lumière ». On peut pas toujours ronchonner, garder tout en dedans, comme font tous les autres. Faut être comme le Survenant : accepter de devenir.

M.P. Dans la scène de l'harmonium, Angélina lit un passage de la Bible (« Marchez dans la lumière afin que vous soyez des enfants de lumière »), ajouté par rapport au roman : pourquoi ce passage ? Quelle est l'importance de la religion pour Angélina ?

E.K. La religion fait partie de son carcan, celui d'une communauté québécoise extrêmement resserrée autour de la religion. Une religion qui a engendré un tort immense dans toutes les communautés francophones, autochtones surtout. Ici, les beaux textes de la religion, venant d'Angélina, sont emprunts de naïveté et de vérité. Citer ce passage revient à garder l'empreinte de ce que nous étions, même si ça ne vient pas forcément dans le texte original. Pour moi, ça permet de vêtir ce personnage-là. À l'époque de l'écriture du roman, c'était quelque chose qui faisait partie de la vie de tous les jours. Au moment du film, plus personne ne va à l'église, plus personne ne prie. Créer un segment qui a ce discours ramène le public à cette époque où des gens basaient leur réalité, la vérité, leur bonheur sur les écrits bibliques, ce qui faisait que les gens étaient pauvres, que les femmes avaient huit enfants... Pour moi, il fallait replacer le récit dans un contexte historique.

M.P. Contrairement à Cadavres [adaptation de François Barcelo réalisée en 2009] où vous avez enlevé la partie où le jeune prêtre se fracasse la tête du haut des marches de la cave...

E.K. Oui, mais par contre, j'ai ajouté le fait que la sœur [Angèle, jouée par Julie Le Breton] devient la Sainte Marie quand elle prie pour sa mère. Je l'ai trafiqué ce moment-là ! On a enlevé le prêtre parce que c'était trop. Ça marchait, narrativement, et j'ai pensé le garder pendant un bout, même que mon frère [Nicolas Canuel] pouvait l'incarner mais ça étirait l'histoire pour rien, c'est pour ça qu'on ne l'a pas fait. Des fois, nécessité oblige, à l'écriture, tu te rends compte que ça ne marche pas.

M.P. Vous avez une séquence où intervient un « cheval mécanique » (une voiture) vers le milieu du film. Peut-on dire que les techniques accumulées (tant au niveau du montage avec le ralenti, qu'au niveau de la bande son) sont l'occasion d'une mise en abyme du conflit entre la modernité (représentée par le Survenant) et le monde rural (représenté par les villageois) ?

E.K. Non, non, tu vas trop loin dans ton analyse. C'est simplement que c'est le genre de séquences qui demande, normalement, énormément de temps pour la rendre aussi dramatique qu'on le souhaite. On l'avait pas ce temps-là donc on s'en est remis à nos connaissances du métier pour trouver un autre moyen pour accentuer le drame, souligner ce qu'on avait à dire dans ce moment, tout en rendant cette scène d'action crédible. Parce que dans une scène d'action comme ça, c'est extrêmement complexe de rendre ça crédible. On avait travaillé longtemps sur la charrette qui revirait en arrière, tombait, glissait sur les gens. C'était bien trop complexe et on s'est fait dire, avec très peu de préavis, qu'il faudrait trouver une autre solution. Qu'est-ce qu'on pouvait faire pour que le langage cinématographique rende le moment crédible et aussi fort que ce qu'on voulait? C'était important que les gens se rendent compte que le Survenant est un gars qui risque sa vie, qui fait acte de sacrifice, pour un enfant d'une communauté avec qui il n'a absolument aucun lien d'attachement. Rajouter ça permet de dire qu'il est prêt à se donner, sous les yeux de tous : soit il devient plus grand que nature, soit encore plus l'homme à abattre. 
M.P. Quel est le rôle des trois séquences axées sur Angélina dans sa chambre ?

E.K. Je voulais lui permettre d'accepter sa féminité, d'accepter que sa difformité ne lui enlève absolument rien, qu'elle reste magnifique sous les yeux de l'univers, de Dieu. Donc ça va un peu avec ce que tu disais tout à l'heure sur l'écrit biblique, quand on parlait de s'approcher de la lumière : je voulais lui dire «ne reste pas cloîtrée, te cache pas, sois belle, sois ce que tu es entièrement ». En plus, c'était accepter sa féminité et sa sexualité à une époque où on empêchait les femmes d'être entières et de prendre leur place. À cette époque, une femme ostracisée par son infirmité est réduite à n'être qu'une monnaie d'échange. Le Survenant amène ce puits de lumière qui traverse les nuages et qui tombe sur tous ces gens. Angélina, devant le miroir, c'est ça.

Mais j'ai aussi dû l'humaniser par rapport au roman, l'actualiser. Même si on est en 2019, la majorité des gens considèrent, encore aujourd'hui et de manière archaïque, que les femmes doivent rester à leur place. C'est un combat de tous les temps et pour moi, il y avait un petit peu de ça aussi, sans le faire à coups de marteau. Dire qu'elle avait ça en elle, le bourgeon est en elle et que le Survenant est juste là pour mettre de l'eau.

La distribution voulait une autre actrice. Mais moi, ça me prenait quelqu'un qui allait prendre le rôle par en-dessous et il n'y avait que deux personnes qui étaient susceptibles de le faire : Suzanne Clément et Anick Lemay. Anick a fait une audition à se lancer sur les murs. J'avais dit aux producteurs et aux distributeurs d'attendre de la voir parce que je croyais en elle parce que je l'avais vue pour $\mathrm{Nez}$ Rouge et elle m'avait enchanté. On l'a choisie avec la bénédiction des producteurs et de la distribution. C'est une femme extraordinaire, d'une sensibilité, d'une sensualité incroyable.

M.P. J'ai l'impression que vous avez trouvé une raison à l'errance du Survenant. Dans trois scènes, il fait en effet référence à son père, qui l'aurait abandonné. Est-il impliqué que le personnage principal a pris la route parce qu'il a été trahi par ses géniteurs ?

E.K. C'est une back-story qui n'est pas soulignée mais je suis content que tu l'aies vu. Ça nous permet d'apprécier le personnage à sa juste valeur et lui donne la chance de dire au fils jaloux ce qu'il a à lui dire, c'est-à-dire qu'il ne se rend pas compte de la chance qu'il a d'avoir une terre et de l'amour qu'on lui donne. Est-ce qu'il est parti à la recherche de son père ? En partie, oui. C'était aussi important d'en faire un personnage ancré émotivement, pas juste un bonimenteur. Dans ce qu'il est et dans la façon dont je l'ai fait évoluer, cette blessure lui donnait une profondeur humaine parce qu'on montre souvent plus qui on est par nos blessures. Et quand on arrive à les comprendre et à passer par-dessus, on devient encore plus généreux, encore plus capable d'aimer les gens et la vie.

M.P. J'ai une dernière question, plus cosmétique, concernant la dernière image du film : je me suis demandée pourquoi il repart du même côté d'où il est venu ?

E.K. Pourquoi la gauche à ton avis ? Ton cœur est de quel bord? Mon père est un homme de théâtre et il disait toujours que quand tu as la chance de te mettre en scène, tu te mets toujours du côté du cœur du public. De la même manière, quand tu racontes l'histoire, ton personnage principal, tu le mets à gauche ou à droite dépendamment de ce que tu veux dire et le public va réagir différemment selon d'où il vient.

\section{Concernant la transcréation/adaptation}

E.K. Les films me choisissent et me donnent les possibilités de mettre mes petits clins d'œil, mes petits moments. C'est sûr qu'il y a des thématiques à travers lesquelles j'essaie de dire symboliquement qui je suis mais, le plus souvent, je me laisse porter. 


\section{M.P. D'après vous, c'est quoi une bonne adaptation ?}

E.K. J'ai fait un film sur Hemingway, un court métrage en Imax, parce qu'Alexandre Petrov avait fait Le vieil homme et la mer en animation avec un peintre russe qui peignait avec les doigts, un cadre à la fois. C'était exceptionnel. Il a gagné un oscar pour ça d'ailleurs. Chez Imax, ils voulaient maintenant un 40 minutes et ils m'ont demandé si ça m'intéresserait. Alors j'ai écrit à partir de la base que les producteurs avaient, un 22 minutes en hommage à Hemingway. L'homme face à lui-même. L'homme face à la nature. Le grand écrivain qui va à la première guerre mondiale. Et on termine sur un pêcheur qui s'en va au loin. J'ai écrit des moments du film comme ça, avec une narration qui lie Hemingway à la grandeur de son âme, à la grandeur de la nature, à la grandeur de l'homme qui doit toujours se dépasser. Et le fils d'Hemingway m'a écrit pour me dire que c'était le plus bel hommage qu'on ait fait de son père. Pour moi, c'était une adaptation de tous ses livres.

Je pense qu'on est comme une guitare ou un piano, un instrument à cordes et quand on lit, on gratte des cordes à certains endroits. Et chacun d'entre nous va interpréter cette mélodie-là différemment parce que c'est pas la même corde qui vibre. On crée tous selon une harmonie qui nous est propre. Pour moi, un film, c'est comme une symphonie, avec ses mouvements, ses crescendos et ses silences. Mon approche est toujours en rapport avec la musicalité que ce soit dans le montage, le choix des plans, les mouvements de caméra. En tant que fan de bande dessinée, je ne peux pas nier qu'il y a une ode, dans la BD moderne, une musicalité que tu retrouves dans le cinéma et dans le roman. Comme si les romans graphiques étaient une charnière entre ces deux univers.

Tout ça m'amène à dire qu'une bonne adaptation revient à ne pas trop intellectualiser l'écrit. Je cherche une lecture qui se veut la plus sobre et la plus émotive possible. Par exemple, ce que j'ai adoré de Cadavres, c'est que ce livre est d'une simplicité incroyable: ça se lit comme tu bois un Coke, tu rotes comme tu rotes après avoir bu un Coke, c'est aussi vicieux, sale, jouissif... et ça a l'intelligence de ne pas se prendre au sérieux tout en étant narrativement une des plus belles embardées que tu puisses avoir dans la lecture. Alors que dans d'autres écrits, comme Le Survenant, c'est pas la raid de la lecture qui va t'enivrer. Quand j'ai lu La loi du cochon, le scénario, je devais faire une série. J'attendais la réponse des producteurs et ils ont retardé un peu trop. Alors j'ai lu le scénario et, après cinq pages, j'ai appelé mon agent pour dire : «C'est ça que je veux faire !» Cadavres, quand je l'ai lu, j'avais la même jouissance. À tous les chapitres, «T'es pas sérieux!», «Ben voyons donc !». Fait qu'une bonne adaptation dépend de ce que le livre provoque en toi. Mais c'est aussi la liberté que tu vas te donner pour en faire ce que tu considères être la plus belle mélodie que cet écrit-là ait pu faire vibrer en toi. Même si c'est pas fidèle aux mots, tant que c'est fidèle à l'inspiration, tant que c'est fidèle à ce que tu te fais toi-même de cette histoirelà, ça marche. Si tu réussis, il y aura des gens qui vont t'écrire et te dire : "J'ai mieux aimé ton film que le roman ». Autant je me suis fait détester pour Cadavres et amouracher pour Cadavres... autant pour d'autres films, j'ai eu ce genre de propos. Comme Lac Mystère [adaptation libre (2013) du roman d'Andrée A. Michaud], je suis complètement allé ailleurs. Dans la deuxième partie du livre, tu tombes dans un délire de résurrection dans le corps d'animaux. Moi, j'ai trouvé que c'était cool. Si j'avais eu les fonds, je l'aurais fait flyé. J'aimais cette espèce d'aura, de mystère et de meurtre, c'est bien bizarre comme film. Je me suis fait dire que ça marchait pas à cause des deux tons, ça commence comme un thriller et ensuite ça tombe dans le registre de la comédie. Il y a des gens pour qui ça marche à fond la caisse pis d'autres pour qui ça fonctionne pas. Dans Lac Mystère, c'était une interprétation libre. Mais l'auteure est venue à la première et avait l'air bien contente. 


\section{M.P. Quel souvenir gardez-vous du Survenant ?}

E.K. C'était un beau tournage. Le directeur photo [Bernard Couture] est formidable. Avec lui, j'ai fait cinq films... six ! J'oubliais Barrymore [adaptation (2011) de la pièce de théâtre de William Luce], avec Christopher Plummer. On s'est fait un beau film, on a eu des beaux prix, des beaux éloges. L'auteur est un gars extraordinaire, d'une douceur et d'une gentillesse mémorables. C'est le fun quand tu rencontres des gens comme ça et que tu passes du bon temps. Christopher a aussi été une perle. Ben le fun. Un grand bonhomme. Une autre époque, une autre façon de vivre. Mais pour Le Survenant, tout le casting était extraordinaire. Par exemple, l'actrice qui joue Phonsine [Catherine Trudeau] est une actrice adorable. Sinon, une séquence dont je suis fier c'est après le départ du Survenant, Angélina est laissée seule avec son père [joué par Germain Houde]. Quand on a fait le montage au début, il y avait beaucoup de dialogues entre le père d'Angélina et elle, qui se berce à la fenêtre où elle a mis la lumière comme on le faisait à l'époque en espérant le retour du mari. J'avais fait un visionnement et Patrick Huard, un copain, et le producteur de Cadavres, avec qui j'avais fait Le dernier tunnel, m'ont tous les deux dit: "As-tu pensé à enlever le dialogue du bonhomme ? La laisser elle, toute seule, regarder dehors ?» C'était ça qui était en trop ! Le père ne pouvait pas être le spectateur et avoir quelque chose à dire. Ce spectateur n'a rien à dire. Donc le père retourne au jardin, la vie continue. C'est plate mais c'est comme ça, car il n'y aura pas de mari pour Angélina.

\section{M.P. Des projets sur lesquels vous travaillez actuellement ?}

E.K. C'est sûr que j'ai un beau métier ! C'est difficile mais c'est un beau métier ! Ça fait un moment que je n'ai pas tourné mais là, on se met à une série TV, ça devrait être intéressant. Je fais ça avec le parrain de mon fils, fait que déjà là, c'est le fun ! C'est pas un show compliqué et j'ai hâte de m'y remettre! Sinon, je développe un film avec le producteur de Cadavres, l'histoire d'un père et de son fils. Un père un peu éclaté qui a abandonné ses enfants et leur mère, et qui est retourné vivre en Angleterre. Son fils essaie de renouer les liens, il invite son père à faire la route 66 . On pense que c'est pour recréer des liens mais finalement, c'est pour le blâmer. On devait le faire en français mais on a décidé de le faire en anglais parce qu'on n'a de financement qu'en anglais. En ce moment, on est dans le casting. Il y a beaucoup de choses oniriques dans le film, beaucoup de visions un peu flyées. C'est un voyage, une belle allégorie, une épopée. Enfin, on verra : peut-être que ça verra le jour, peut-être pas... 